\title{
Study on Simulation for Vehicle Ride Comfort Based on MATLAB
}

\author{
Fengping $\mathrm{CaO}^{1, \mathrm{a}}$, ,Chunmei Chen ${ }^{2, \mathrm{~b}}$ and Lifa Zhou ${ }^{3, \mathrm{c}}$ \\ ${ }^{1}$ School of Shandong Jiaotong University, Jinan 250357, China. \\ ${ }^{2}$ School of Shandong Transport Vocational Collage, Weifang 261206, China. \\ ${ }^{3}$ Sinotruk (Hong Kong) Limited South Asia \& America Area, Jinan 250101, China \\ acao2138@163.com, bailing_mm@163.com, czhoulf@sinotruk.com
}

Keywords: Automobile ride comfort, Matlab/Simlink, Simulation analysis

\begin{abstract}
Ride comfort is an important performance index of a automobile, and it directly affects people's riding comfort. Suspension system is an important factor affecting the ride comfort of the car. In the paper, the influence of suspension on vehicle ride comfort was analyzed, then a model of automobile suspension system based on Matlab/Simulink was established to simulate the ride comfort of vehicles. The study provided a research method for analyzing the impact of suspension system on vehicle ride comfort, and also provided reference for optimization of suspension parameters during vehicle design.
\end{abstract}

\section{Introduction}

With the development of society and economy, people's living standards have been continuously improved, and there is a higher requirement for the comfort of the car's riding environment[1]. To ensure that the car's vibration is within a certain range, so that the driver can have good psychological and physiological conditions under long-term driving conditions. Excellent ride comfort is not only the guarantee to improve the riding comfort, but also important to the safe driving[2]. In the paper, the effect of suspension system on ride comfort based on Matlab was simulated and analyzed, and it can provide a research method for optimizing suspension parameters during vehicle design.

\section{Effect of Suspension System on Vehicle Ride Comfort}

The vehicle suspension system is generally consisted of spring, damper, oriented framework and anti-roll bar. Suspension is divided into two categories according to the control form: passive suspension and active suspension[3].

Suspension Stiffness. Suspension stiffness determines the natural frequency of the suspension system. The car has high requirements for ride comfort, and usually chooses a relatively low body natural frequency to obtain smaller acceleration. The general frequency range of a car is within $1 \sim 1.5 \mathrm{~Hz}$. On the contrary, off-road vehicles and trucks usually have poor road conditions, and a relatively high natural body frequency must be selected. Generally, the frequency range selected is 1.5 to $2 \mathrm{~Hz}$.

Suspension Static Deflection. Suspension static deflection directly affects the body natural frequency. When the elastic properties of the suspension used are linear changes, the static deflection of the front suspension $f_{c_{1}}$ can be expressed as follows.

$$
f_{c_{1}}=m_{1} g / k_{1}
$$

Equation (2) is the static deflection of the rear suspension $f_{c_{2}}$.

$$
f_{c_{2}}=m_{2} g / k_{2}
$$

Where $k_{1}, k_{2}$ are the suspension stiffness of front and rear suspension respectively, $m_{1}, m_{2}$ are the sprung mass of front and rear suspension respectively and $g$ is the gravitational acceleration [3]. 
Unsprung Mass. When the unsprung mass is large, the impact load received by the suspension system and transmitted to the vehicle body is also large. When the unsprung mass of the independent suspension is small, the impact and vibration from the road surface can reduced and the ride comfort of the car improved. The effect of unsprung mass on ride comfort is often evaluated by the ratio of unsprung mass to sprung mass. The smaller the value, the better.

Relative Damping Ratio. When the vehicle suspension has only elastic elements and no vibration damping device, the vibration of the vehicle suspension mass will continue for a long time, making the passenger feel uncomfortable. Therefore, there must be damping force in the suspension to damp vibration. After the suspension stiffness is determined, the damping force must be properly selected in order to give full play to the damping effect of the suspension. When the damper of the suspension system is linear, the speed of vibration attenuation can be evaluated by the relative damping ratio. The expression is shown as follows[4].

$$
\zeta=\frac{c}{2 \sqrt{k m}}
$$

Where $c$ is the equivalent damping coefficient of the suspension damper, $k$ is the suspension stiffness and $m$ is the suspension mass.

\section{System Model Based on Matlab}

Road Excitation Model. Road roughness is often used to describe the level of road surface undulations and is the main incentive for the car to drive. The filter white noise that is relatively easy to implement is used as the road surface input model, which is shown as follows [2].

$$
q^{\prime}(t)=-2 \pi f_{0} q(t)+2 \pi \sqrt{G_{0} u_{0}} w(t)
$$

Where $q(t)$ is the pavement displacement, $G_{0}$ is the Road roughness coefficient, $u_{0}$ is the vehicle speed, $w(t)$ is the white noise with a mean of zero and $f_{0}$ is the lower frequency cut-off.

Fig.1 is the established simulation model of road excitation based on Matlab/simulink[5].

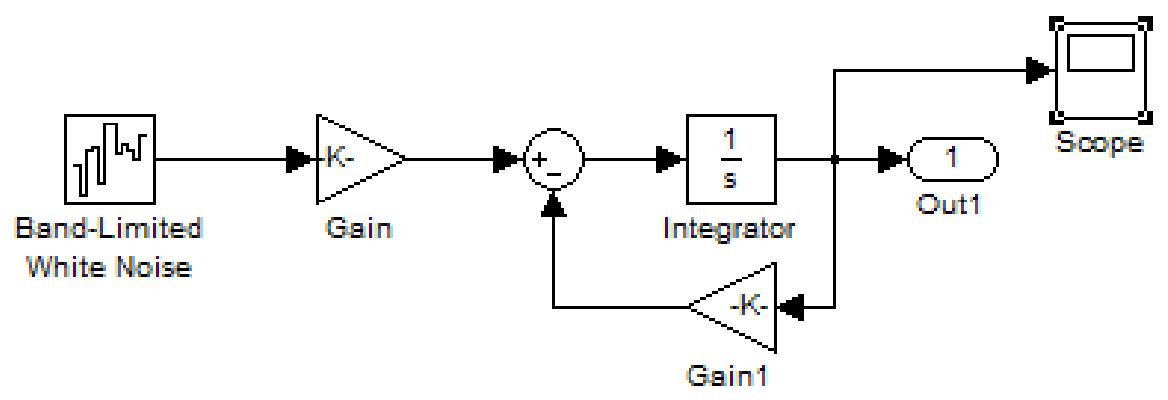

Fig.1. Simulation model of road excitation

Passive Suspension System Model. The automobile is a more complex vibration system and should be simplified according to the problem to be analyzed.

The automotive vibration system can usually be reduced to a single-mass system and a dual-mass system. Because the dual-mass system can not only reflect the dynamic characteristics of the body part, but also reflect the dynamic characteristics of the wheel part in the generation of high-frequency resonance, it is closer to the actual situation of the automotive suspension system. Therefore, the general automotive vibration system is reduced to a dual-quality system. Fig. 2 is the model of a two-degree-of-freedom vibration system for a passive suspension, where $\mathrm{Z}_{1}, \mathrm{Z}_{2}$ is the vertical displacement of the wheel and body respectively, $q$ is the input road roughness function, $m_{2}$ is the sprung mass, $m_{1}$ is the unsprung mass, $K$ is the suspension stiffness, $c$ is the damping coefficient, and $K_{t}$ is the tire stiffness. 


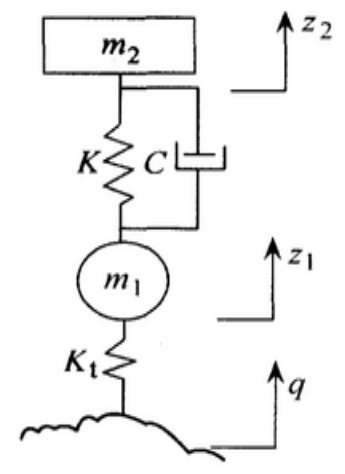

Fig. 2. Two-degree-of-freedom vibration system model of passive suspension

The coordinates are selected at their respective equilibrium positions, then the differential equation can be described as follows[6].

$$
\left\{\begin{array}{l}
m_{1} z_{1}=K\left(z_{2}-z_{1}\right)+C\left(z_{2}-z_{1}\right)-k_{t}\left(z_{1}-q\right) \\
m_{2} z_{2}=K\left(z_{1}-z_{2}\right)+C\left(z_{1}-z_{2}\right)
\end{array}\right.
$$

According to the above physical model and differential equations, a passive suspension system simulation model is established based on Matlab/Simulink, which is shown as Fig.3.

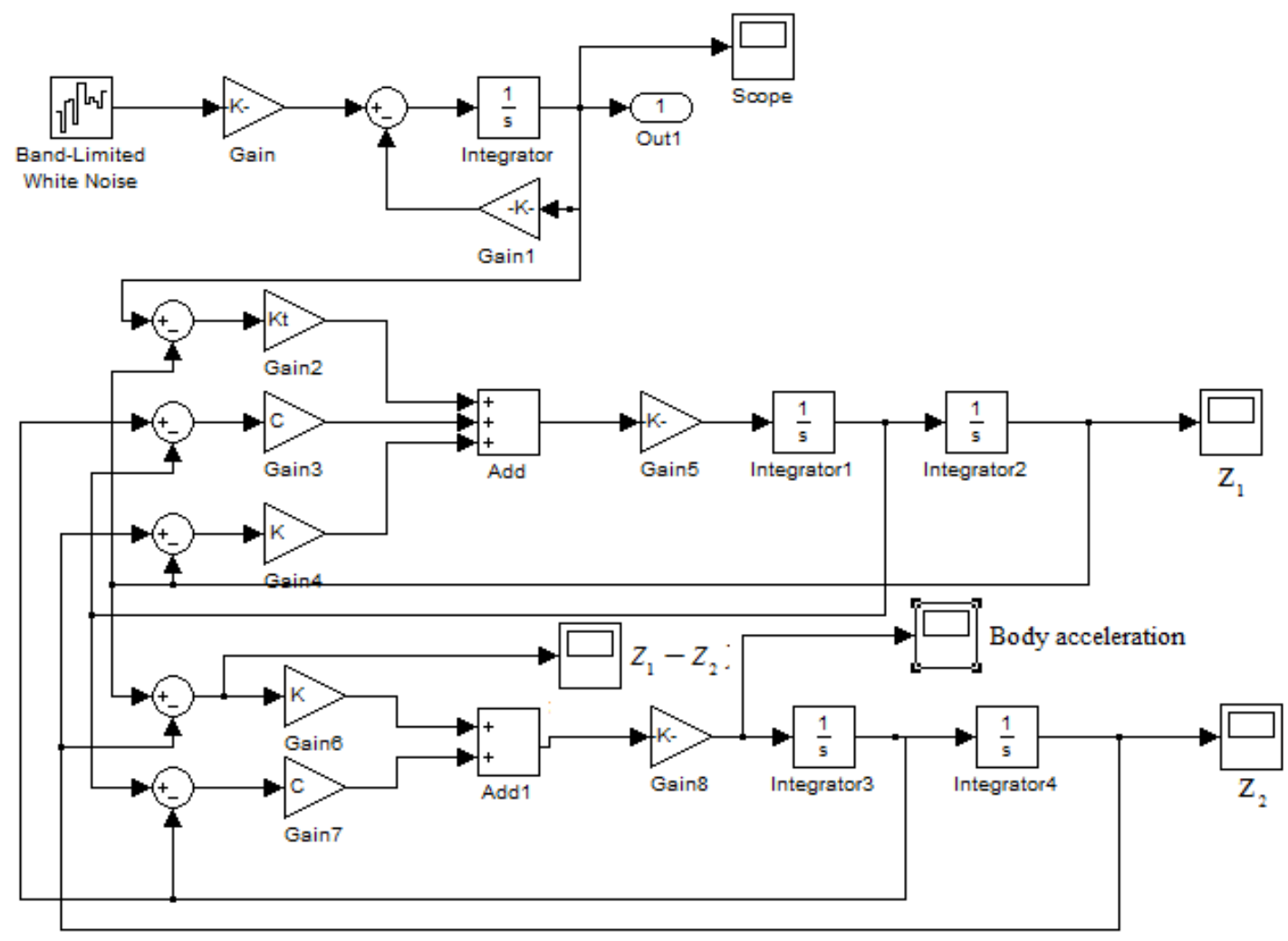

Fig.3. Passive suspension system simulation model

Active Suspension System Model. The active suspension can overcome the defects of the passive suspension. It adopts passive or active control components and is a closed-loop control system. According to the road conditions and the running status of the vehicle, the electronic control unit ECU performs real-time calculations, then issue corresponding instructions to the damper controller, take the initiative to react, control the vehicle suspension parameters, so the damping of the suspension is always optimal [11].

The two-degree-of-freedom vibration system model of active suspension is shown in Fig.4. The system motion status signal is input to the ECU through the sensor, and the ECU issues an instruction to the force generator after analysis to generate an active control force to adjust the characteristics of the suspension system. 


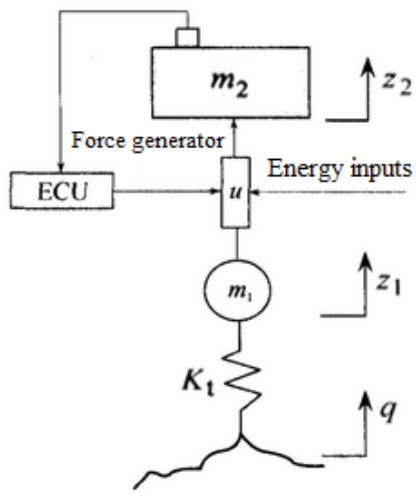

Fig.4. Two-degree-of-freedom vibration system model of active suspension

In Fig.4, $l_{1}, l_{2}, l_{3}$ are the feedback coefficients obtained according to the optimization; other parameters are the same as the passive suspension system, $u$ is the active control force, and it can be described as follows.

$$
u=-\left(l_{1}\left(z_{2}-z_{1}\right)+l_{2} z_{1}+l_{3} z_{2}\right)
$$

The equation of motion can be expressed as follows.

$$
\left\{\begin{array}{l}
m_{1} z_{1}=l_{1}\left(z_{2}-z_{1}\right)+l_{2} z_{1}+l_{3} z_{2}-k_{t}\left(z_{1}-q\right) \\
m_{2} z_{2}-l_{1}\left(z_{2}-z_{1}\right)-l_{2} z_{1}-l_{3} z_{2}
\end{array}\right.
$$

According to the above physical model and motion equations, the active suspension system simulation model established based on Matlab/Simulink is shown as Fig.5.

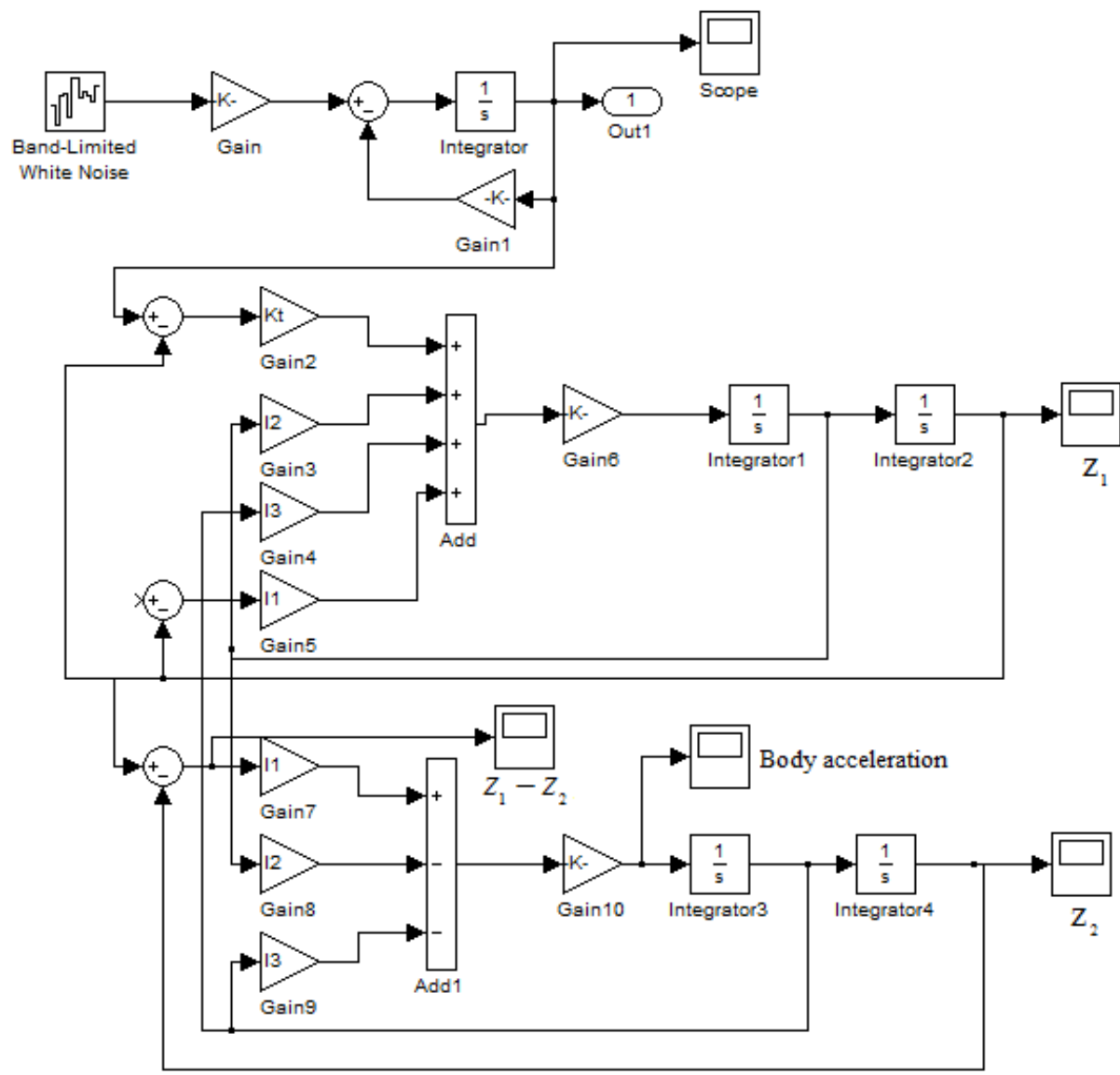

Fig.5. Passive suspension system simulation model 


\section{Simulation Experiment}

Time-domain Simulation of Road Excitation. The simulation results are shown in Fig.6 while the values of the parameters are $f_{0}=0.1 \mathrm{~Hz}, G_{0}=64, u_{0}=20 \mathrm{~m} / \mathrm{s}$.

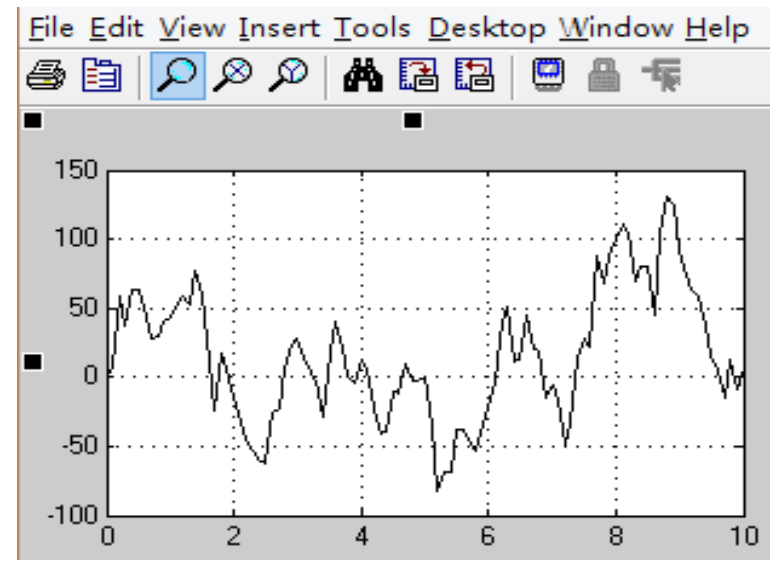

Fig.6. Simulation results of road excitation

Simulation of Suspension System. For the passive suspension system simulation model in Fig. 4, the parameters are as follows: $m_{1}=24 \mathrm{~kg}, m_{2}=240 \mathrm{~kg}, K=9475 \mathrm{~N} / \mathrm{m}, K_{t}=85270 \mathrm{~N} / \mathrm{m}$, $c=754 \mathrm{~N} \cdot \mathrm{m} / \mathrm{s}$. While for the active suspension system simulation model, the feedback coefficients are as follows: $l_{1}=7592 \mathrm{~N} / \mathrm{m}, l_{2}=481 \mathrm{~N} \cdot \mathrm{s} / \mathrm{m}, l_{3}=1916 \mathrm{~N} \cdot \mathrm{s} / \mathrm{m}$. The simulation results of body displacement, body acceleration, and suspension travel are shown as Fig.7, where the solid line represent the simulation results of the active suspension, and the dashed line represents the passive suspension.

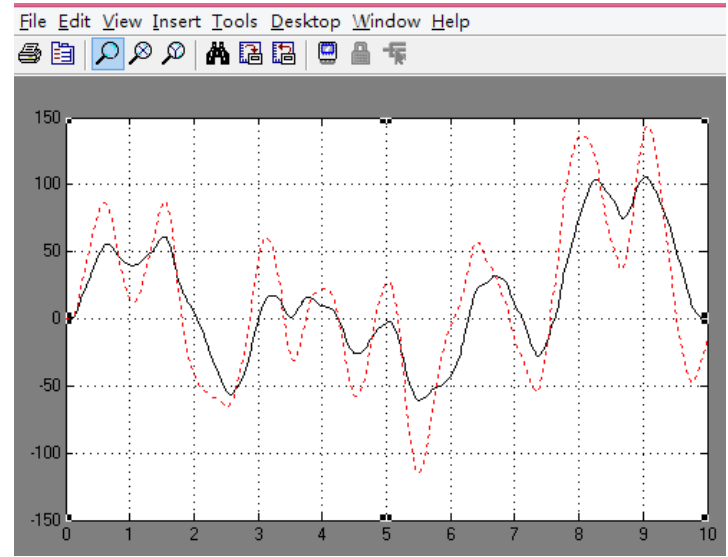

(a) Body displacement

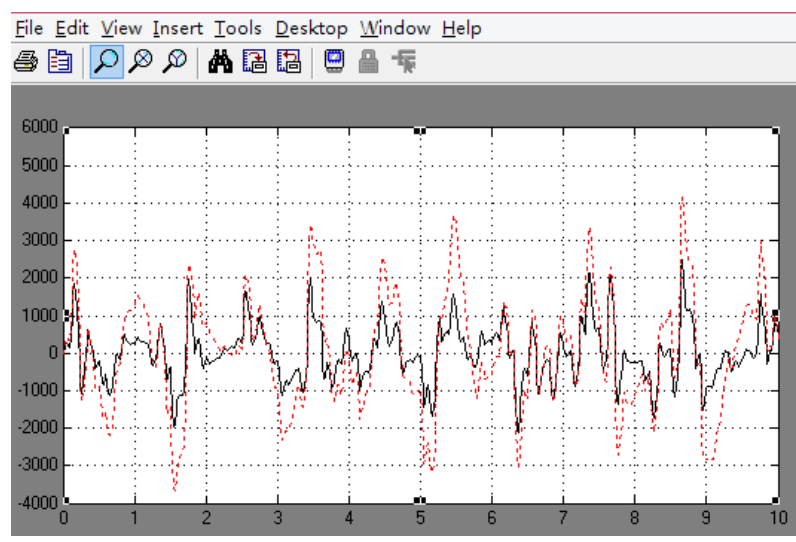

(b)Body acceleration

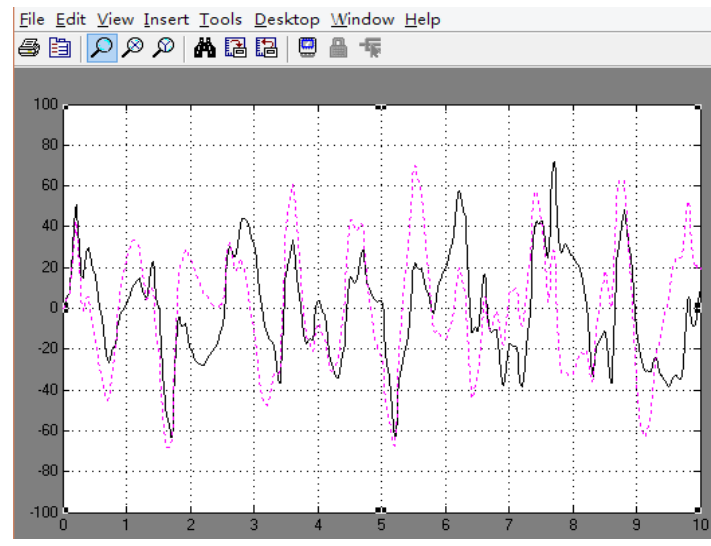

(c)Suspension travel

Fig.7. Comparison of simulation results of the passive and active suspension 
Fig. 7 (a) indicates that the active suspension has smaller amplitude than passive suspension under the same road surface roughness excitation. It can be seen from Fig. 7 (b) that the body acceleration of the active suspension is smaller than that of the passive suspension, which indicate that the active suspension can attenuate the vibration better. It can be seen from Fig. 7 (c) that the active suspension travel is smaller than the passive suspension, but the difference is significant. This is related to the selection of the active suspension feedback coefficient. In the specific design, the optimal value can be selected by optimizing the design to achieve the desired effect.

\section{Conclusions}

In the paper, a simulation algorithm of automobile ride comfort based on Matlab is presented. Firstly, the influence of automobile suspension system on vehicle ride comfort is analyzed, including suspension stiffness, suspension static deflection, unsprung mass and relative damping ratio. Then, the road excitation model, passive and active suspension system models were established respectively. The simulation were executed using Matlab/Simulink. The simulation results show that the active suspension can dynamically adjust the stiffness and damping, so that the car can better absorb the impact and attenuate the vibration in the driving process, thus showing a better ride comfort.

\section{Acknowledgements}

This research is supported by Natural Science Foundation of Shandong Province (ZR2015EL027), thanks to Natural Science Foundation committee of Shandong Province.

\section{References}

[1] http://www.ocn.com.cn/qiche/201801/hhhfa02174029.shtml

[2] Yu Zhisheng. Automobile theory[M].Beijing: China Machine Press, 2009. (in Chinese)

[3] Yu Fan. Automotive System Dynamics. Beijing: Machinery Industry Press, 2005.(in Chinese)

[4] Zhou Changcheng. Automotive Ride and Suspension System Design. Beijing: Machinery Industry Press, 2011.(in Chinese)

[5] Li Ying. The basic system modeling and simulation of Simulink. Xi'an: Xidian University Press, 2004. (in Chinese)

[6] Li Yida. Control System Design and Simulation. Beijing: Qinghua University Press, 2009. (in Chinese) 